\title{
Association between Changes in Cortical Thickness and Functional Connectivity in Male Patients with Alcohol-dependence
}

\author{
Shin-Eui Park ${ }^{1}$, Yeong-Jae Jeon ${ }^{2}$ and Hyeon-Man Baek ${ }^{1,2 *}$ \\ ${ }^{1}$ Lee Gil Ya Cancer \& Diabetes Institute, Gachon University, Incheon 21999, ${ }^{2}$ Department of Health Science and Technology, \\ GAIHST, Gachon University, Incheon 21936, Korea
}

\begin{abstract}
Many studies have reported structural or functional brain changes in patients with alcohol-dependence (ADPs). However, there has been an insufficient number of studies that were able to identify functional changes along with structural abnormalities in ADPs. Since neuronal cell death can lead to abnormal brain function, a multimodal approach combined with structural and functional studies is necessary to understand definitive neural mechanisms. Here, we explored regional difference in cortical thickness and their impact on functional connection along with clinical relevance. Fifteen male ADPs who have been diagnosed by the Diagnostic and Statistical Manual of Mental Disorders 5 (DSM-5) underwent highresolution T1 and resting-state functional magnetic resonance imaging (MRI) scans together with 15 male healthy controls (HCs). The acquired MRI data were post-processed using the Computational Anatomy Toolbox (CAT 12) and CONN-fMRI functional connectivity (FC) toolbox with Statistical Parametric Mapping (SPM 12). When compared with male HCs, the male ADPs showed significantly reduced cortical thickness in the left postcentral gyrus (PoCG), an area responsible for altered resting-state FC patterns in male ADPs. Statistically higher FCs in PoCG-cerebellum (Cb) and lower FCs in PoCG-supplementary motor area (SMA) were observed in male ADPs. In particular, the FCs with PoCG-Cb positively correlated with alcohol use disorders identification test (AUDIT) scores in male ADPs. Our findings suggest that the association of brain structural abnormalities and FC changes could be a characteristic difference in male ADPs. These findings can be useful in understanding the neural mechanisms associated with anatomical, functional and clinical features of individuals with alcoholism.
\end{abstract}

Key words: Male patients with alcohol-dependence, Alcoholism, Brain cortical thickness, Postcentral gyrus, Resting-state functional connectivity

\section{INTRODUCTION}

Alcohol consumption, abuse and addiction have been responsible for major health, social and economic problems worldwide [13]. Treatments for alcoholism are characterized by poor treatment outcomes and high relapse rates [3]. Treatment failures for alcoholics can lead to motivational loss and worsened personal and interpersonal health [4], which in turn can lead to brain disorders from

Submitted October 1, 2021, Revised November 22, 2021,

Accepted November 22, 2021

* To whom correspondence should be addressed. TEL: 82-32-899-6678, FAX: 82-32-899-6677

e-mail:hmbaek98@gachon.ac.kr changes in brain structure and function [5]. The intake of alcohol affects a person's brain from the bloodstream. When an individual consumes alcohol, the ethanol from the alcoholic drink travels to the brain through the bloodstream, affecting various neuronal functions temporarily through enhanced effects of neurotransmitters along with toxic byproducts. Effects from alcohol are typically progressively weakened in a healthy person as the liver quickly filters out alcohol from the bloodstream, preventing alcohol from further affecting the brain. However, when a person drinks excessively, alcohol from the body is not filtered out fast enough, prolonging the toxic effects of alcohol in the brain. Damage to the brain is mainly mediated by a neurotoxic environment stemming from increased oxidative stress and proinflammatory cytokines [6].
Copyright (c) Experimental Neurobiology 2021. www.enjournal.org
This is an Open Access article distributed under the terms of the Creative Commons Attribution Non-Commercial License (http://creativecommons.org/licenses/by-nc/4.0) which permits unrestricted non-commercial use, distribution, and reproduction in any medium, provided the original work is properly cited. 
Therefore, understanding the brain abnormalities related to neural mechanisms in alcohol-dependent patients (ADPs) is very important in improving clinical practice. Neuroimaging has been widely used to investigate the consequences of alcoholism including atrophy of the gray $[5,7,8]$ and white matters (GM/WM) [9], sulcal widening [10], decreased cortical thickness [11] and ventricular enlargement [10].

Most previous neuroanatomical studies dealing with alcoholism have focused somewhat on WM rather than GM. More specifically, the ADPs showed shrinkages of WM in various cortical regions including the precentral gyrus, anterior cingulate cortex, temporal lobe [12, 13], and anterior hippocampal region [14]. However, studies have also shown that GM structural components are directly associated with functional components $[15,16]$. Recent studies $[5,17]$ showed that structural abnormalities of GM are associated with abnormal functional connections within neural networks of relevant brain regions in ADPs. These results could be evidence that altered functional connectivity (FC) is influenced by GM structural changes due to alcoholism. Until now, multimodal neuroimaging studies that comprehensively examine the changes in the brain associated with synchronous neurofunctional and structural abnormalities in ADPs are still lacking.

Voxel- or surface-based morphology measurement (VBM/SBM) methods are commonly used to analyze GM morphology changes using MRI data. While VBM can only measure brain volumes in voxel units, SBM is capable of probing various aspects of cortical shape including cortical thickness, degree of gyrification, and sulcal depth, providing more insight than VBM [18]. Moreover, to our knowledge, there are no previous studies that have investigated structural and FC changes in ADPs using SBM until now.

In addition, there are gender-related differences in the susceptibility, progression and clinical outcomes of alcohol dependence. For instance, women consume less alcohol, start drinking later and have lower rates of alcohol dependence than men [19]. However, studies have shown that women transition from first alcohol use to alcohol dependence more rapidly than men [20]. Therefore, in addition to gender-related neuroanatomical differences reported by a recent neuroimaging study [21], only male subjects were selected in consideration of gender differences.

Given the above, we hypothesized that there are key regions that have distinct neuroanatomical differences, and affect the brain functional network in male ADPs. Here, we performed a multimodal study employing SBM and resting-state fMRI (rs-fMRI) analysis to investigate distinct brain alterations such as cortical shape including cortical thickness, degree of gyrification, and sulcal depth across entire brain areas as well as their correlation with FCs in male ADPs.

\section{MATERIALS AND METHODS}

\section{Subjects}

Fifteen male ADPs (mean age, $47.53 \pm 6.51$ years) participated in this study along with 15 healthy controls (HCs) (mean age, $47.60 \pm 3.91$ years) (Table 1). A psychiatrist diagnosed their alcoholism using the DSM-5 criteria. The Institutional Review Board of Chungbuk National University Hospital (IRB-CNUH) in Korea approved this study protocol. Before MRI scanning, all volunteers received an explanation of all the experimental procedures and provided written informed consent. Also, all the experimental procedures and methods were performed in accordance with the relevant guidelines and regulations approved by the IRB-CNUH.

\section{Inclusion criteria}

a. DSM- 5 criteria for ADPs

b. Male patients between 20 and 60 years of age.

c. All participant have an education above middle school (more than 9 years: middle school in Korea).

d. Right-handed.

Table 1. Demographic and clinical characteristics between male patients with alcohol-dependence (ADPs) and male healthy controls (HCs)

\begin{tabular}{lccc}
\hline & Alcohol-depent patients $(\mathbf{n}=\mathbf{1 5})$ & Healthy controls $(\mathbf{n}=\mathbf{1 5})$ & $\mathbf{p}$-value \\
\hline Mean are (years) & $47.53 \pm 6.51$ & $47.60 \pm 3.91$ & $\mathrm{p}=0.902^{*}$ \\
Gender (male/female) & $15 / 0$ & $15 / 0$ & $\mathrm{p}=1.000^{* *}$ \\
Handedness (right: left: mixed) & $15: 0: 0$ & $15: 0: 0$ & $\mathrm{p}=1.000^{* *}$ \\
Education (years) & $12.27 \pm 2.71$ & $14.53 \pm 2.20$ & $\mathrm{p}=0.023^{\star}$ \\
Duration of smoking (years) & $26.87 \pm 9.25$ & $16.87 \pm 12.69$ & $\mathrm{p}=0.015^{*}$ \\
Clinical rating scales & & & \\
Duration of drinking per week (days) & $4.73 \pm 2.05$ & $1.57 \pm 1.79$ & $\mathrm{p}<0.000^{*}$ \\
BMAST & $22.27 \pm 5.36$ & $2.07 \pm 3.59$ & $\mathrm{p}<0.000^{*}$ \\
AUDIT-K & $31.47 \pm 7.17$ & $11.87 \pm 6.83$ & $\mathrm{p}<0.000^{*}$ \\
\hline
\end{tabular}

${ }^{\star}$ Mann-Whitney U-test. ${ }^{* *}$ Chi square test.

AUDIT-K: Alcohol Use Disorder Identification Test-Korea, BMAST: Brief Michigan Alcoholism Screening Test. 


\section{Exclusion criteria}

a. Current or past psychiatric illness other than ADPs - dementia, delirium and other organic disorders, mental retardation, psychotic disorders such as schizophrenia, delusional disorder and others, mood disorders, and anxiety disorders.

b. Substance use other than alcohol and tobacco.

c. History of head trauma.

d. History of cardiovascular or endocrine disease.

e. Current medical illness.

f. Presence of magnetically active object in the body.

\section{Magnetic resonance imaging acquisition}

All the subjects underwent MRI with a 3 Tesla Philips Achieva scanner equipped with an 8-channel head coil. The high resolution T1-weighted images were acquired using a three dimensional Turbo Field Echo (TFE) sequence with repetition time/echo time $(\mathrm{TR} / \mathrm{TE})=6.8 / 3.1 \mathrm{~ms}$, flip angle $=9^{\circ}$, field-of-view $(\mathrm{FOV})=288 \times 288$ $\mathrm{cm}^{2}$, matrix size $=256 \times 256$, number of excitation $(\mathrm{NEX})=1$, slice thickness $=1.2 \mathrm{~mm}$ and slices $=170$.

Functional images were acquired using a gradient echo-echo planner image (GRE-EPI) with the following parameters: TR/ $\mathrm{TE}=3,000 / 30 \mathrm{~ms}$, flip angle $=80^{\circ}, \mathrm{FOV}=22 \times 22 \mathrm{~cm}^{2}$, matrix size $=64 \times 64, \mathrm{NEX}=1$, number of slices $=48,3 \mathrm{~mm}$ isotropic voxels and total scan time $=7 \mathrm{~min}$.

\section{Data pre-processing and cortical surface extraction}

The high-resolution T1 images were pre-processed using an automated program in the Computational Anatomy Toolbox (CAT12) based on Matrix Laboratory (MATLAB) (R2016b; MathWorks, Natick, MA, USA) to generate a cortical surface that provided cortical thickness measurement. All of the individual data were processed as follows: 1) bias-field correction, 2) alignment with the anterior and posterior commissures line on the sagittal plane and skull dissection, 3) alignment with the Montreal Neurological Institute standard template (MNI-152), 4) segmentation into GM, WM, and cerebrospinal fluid (CSF) using tissue probability maps based on the International Consortium of Brain Mapping (ICBM) template for East Asian brains, and 5) smoothing with a $15 \mathrm{~mm}$ full-width at half-maximum (FWHM) isotropic Gaussian kernel for cortical thickness and $20 \mathrm{~mm}$ for gyrification index and sulcal depth. The smoothed images were used for between group analyses. Additionally, the total intracranial volume (TIV) was calculated for comparing the brain volume difference in the two groups. The cortical thickness, which is the distance between GM and WM surfaces (approximately 1.6 4.5 mm), was calculated using a fully automated method based on the projection-based thickness (PBT) measurement [22].
Between-group differences in cortical thickness, gyrification index, and sulcal depth were assessed with two-sample t-test controlling for smoking and education level. Statistical maps were thresholded at uncorrected $\mathrm{p}<0.001$ (more than 70 voxels).

The clusters of significant differences in cortical thickness, gyrification index and sulcal depth were considered to be the seeds for further FC analysis.

\section{FC analysis}

FC analysis was performed by using the CONN-fMRI FC toolbox (ver.17e) with Statistical Parametric Mapping-12 (SPM12) as follows: slice timing correction (interleaved), realignment (subject-motion threshold: $2 \mathrm{~mm}$ ), and co-registration. The coregistered images were segmented into the GM, WM, and CSF using standard SPM tissue probability maps. These images were then smoothed with an $8 \mathrm{~mm}$ FWHM Gaussian kernel. For optimal functional outlier detection, the liberal setting with 97 th percentiles in normative sample and global-signal z-value threshold $=9$ was adopted. Unwanted noises of blood-oxygenation-leveldependent (BOLD) signals such as motion, physiologic, and other artifacts were reduced through the use of an anatomical component-based noise correction method (aCompCor) [23]. Band-pass filtering was performed with a frequency window of $0.01 \sim 0.1 \mathrm{~Hz}$. Smoking and education level were controlled as covariates.

For the evaluation of FC, a region of interest (ROI)-to-voxel FC analytical method was used. Here, we specified a spherical cluster with $10 \mathrm{~mm}$ diameters and peak coordinates as the ROI in the left postcentral gyrus (PoCG) (MNI coordination: $\mathrm{x}=-48, \mathrm{y}=-12, \mathrm{z}=31$ ) based on the brain cortical thickness results. Significant connections were identified by two sample t-test calculating as voxel threshold: uncorr. $\mathrm{p}<0.001$, cluster threshold: $\mathrm{p}-\mathrm{FDR}<0.05$.

\section{Statistical analysis of clinical measurements and FC}

To analyze the correlation between brain FC and alcoholism severity, Spearman's correlation coefficient test was performed using the Statistical Package for the Social Sciences (SPSS) statistical software package (version 20.0, SPSS Inc., Chicago, USA).

\section{RESULTS}

\section{Demographic and clinical measurements}

There were no differences among the subjects male ADPs and male HCs in terms of age, handedness and gender distribution ( $p>0.05)$, but length of education $(\mathrm{p}=0.023)$ and duration of smoking $(p=0.015)$ were statistically different (Table 1$)$. The duration of drinking per week in male ADPs and male HCs was 4.73 \pm 2.05 $($ mean \pm SD) and $1.57 \pm 1.79$ day, respectively. Compared to male 
Table 2. Comparison of brain volumes between male patients with alcohol-dependence (ADPs) and male healthy controls (HCs)

\begin{tabular}{lccc} 
& ADPs & HCs & p-value \\
\hline Gray matter volumes $(\mathrm{ml})$ & $673.59 \pm 71.27$ & $675.80 \pm 43.82$ & $\mathrm{p}=0.820^{*}$ \\
White matter volumes $(\mathrm{ml})$ & $526.75 \pm 57.54$ & $556.96 \pm 59.60$ & $\mathrm{p}=0.290^{*}$ \\
CerebroSpinal fluid $(\mathrm{ml})$ & $351.32 \pm 71.39$ & $324.69 \pm 53.30$ & $\mathrm{p}=0.221^{*}$ \\
Total crenial volumes $(\mathrm{ml})$ & $1,551.66 \pm 148.47$ & $1,557.45 \pm 124.41$ & $\mathrm{p}=0.917^{*}$ \\
\hline
\end{tabular}

${ }^{\star}$ Mann-Whitney U-test.

HCs, male ADPs showed higher scores in the Brief Michigan Alcohol screening test (BMAST) and alcohol use disorders identification test (AUDIT) $(\mathrm{p}<0.000)$ (Table 1). The mean scores for BMAST and AUDIT of the male ADPs were 22.27 \pm 2.71 and $31.47 \pm 7.17$, respectively (Table 1 ).

\section{Comparison of brain volumes}

The GM volumes of male ADPs and male HCs were $673.59 \pm 71.27 \mathrm{ml}$ and $675.80 \pm 43.82 \mathrm{ml}$, respectively $(\mathrm{p}=0.820)$, whereas their $\mathrm{WM}$ volumes were $526.75 \pm 57.54 \mathrm{ml}$ and $556.96 \pm 59.60 \mathrm{ml}$, respectively $(\mathrm{p}=0.290)$. There were no statistically significant differences in total brain volumes (TIV: $\mathrm{GM}+\mathrm{WM}+\mathrm{CSF}$ ) between the two groups with $1551.66 \pm 148.47 \mathrm{ml}$ and $1557.45 \pm 124.41 \mathrm{ml}(\mathrm{p}=0.917)$ (Table 2).

\section{Cortical thickness reduction in male ADPs}

Significant differences in gyrification index and sulcal depth between the two groups were not observed.

In cortical thickness, Fig. 1 shows patterns of mean cerebral cortical thickness for male HCs (Fig. 1A) and male ADPs (Fig. 1B) (uncorr. $\mathrm{p}<0.001$ ). Compared to male HCs, the male ADPs showed decreased cortical surface thickness in the left postcentral gyrus (PoCG) (uncorr. $\mathrm{p}<0.001$, cluster size: 74 voxels) (Fig. 2). However, there was no increased cortical surface thickness in patients.

\section{Differentiated FC between male ADPs and male HCs}

The results of FC using seed-to-voxel analysis between male ADPs and male HCs are shown in Fig. 3, 4 and Table 3. In the comparison of FCs with and without structural results, large FC patterns were observed in the whole left PoCG rather than strictly at the localized PoCG originating from coordinates linked with reduced cortical thickness. (voxel level uncorr. $\mathrm{p}<0.001$, uncorrected; cluster level $\mathrm{p}<0.05$, FDR corrected) (Fig. 3).

There were similar FC patterns in both groups. The male ADPs showed PoCG with a large FC cluster extending frontally, but relatively weaker and more restricted. Additionally, male HCs showed FCs linked with the inferior and middle occipital gyrus, lingual gyrus, and fusiform gyrus.
In the group comparison (Fig. 4), there was no significant difference between the two groups in the results of whole left PoCG. On the other hand, distinct differences were observed in localized PoCG which showed reduced cortical thickness.

Significantly lower FC in the supplementary motor area (SMA) (Fig. 4A) and higher FC in the cerebellum (Cb) (Fig. 4B) were observed in male ADPs compared to male HCs (Table 4) (two sample $\mathrm{t}$-test, voxel level uncorr. $\mathrm{p}<0.001$, uncorrected; cluster level $\mathrm{p}<0.05$, FDR corrected).

\section{Correlation between FC and alcoholism severity}

Fig. 4 shows a significantly positive correlation between AUDIT scores and FC of $\mathrm{Cb}$ linked to PoCG where there was reduced local cortical thickness in male ADPs Spearman's correlation $(\mathrm{r})=0.579, \mathrm{p}=0.024)$ (Fig. 5). However, there was no significant correlation with BMAST.

\section{DISCUSSION}

In this study, we applied a multimodal approach to assess whether if there were brain structural alterations causing functional abnormalities in male ADPs, and subsequently assess any correlations between alterations in brain structures and clinical severity. Here, we hypothesized that there are distinct brain structural and functional alteration patterns that are affected by alcoholism in the male ADPs. In addition, these findings can provide valuable knowledge regarding abnormalities in neural pathways and other related pathophysiological mechanisms that can occur in male ADPs.

ADPs had significantly lower levels of education and smoking duration compared to HC in our study. To account for their influences on significant brain structure alterations, education level and smoking duration were set as coefficients for significant groups analysis. A previous study [5] evaluated potential effects of education on functional and structural results by comparing brain structures of cohorts with and without matching education years. The results showed that the patterns of rs-FC and structural difference were very similar between the two groups. Also, several studies 

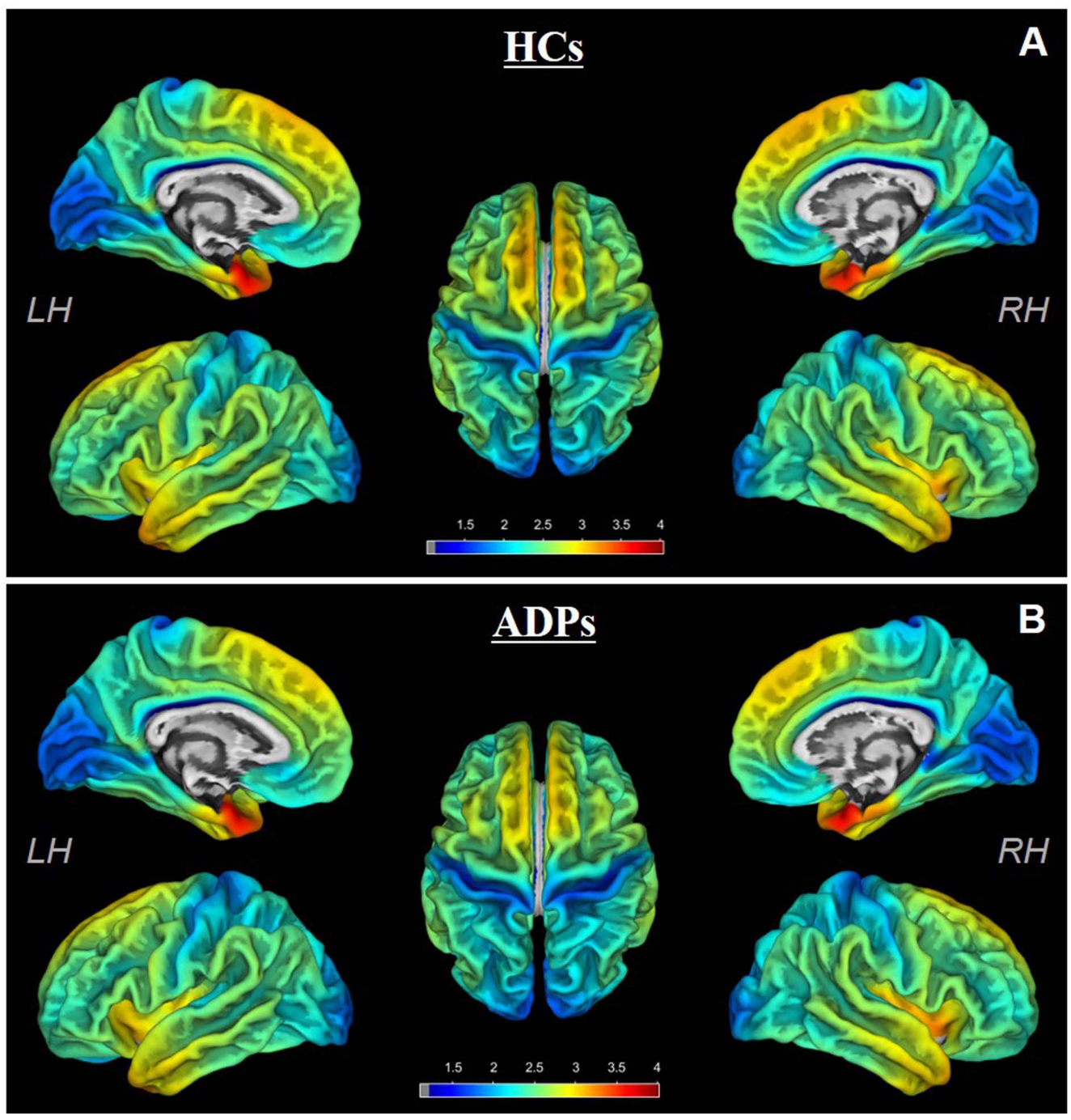

Fig. 1. Cerebral thickness patterns in male healthy controls (HCs) (A) and male patients with Alcohol-dependence (ADPs) (B) (one smaple t-test, uncorr. $\mathrm{p}<0.001)$. The color bar indicates thickness degree (thinner: blue thicker: red). LH, left hemisphere; $\mathrm{RH}$, right hemisphere.

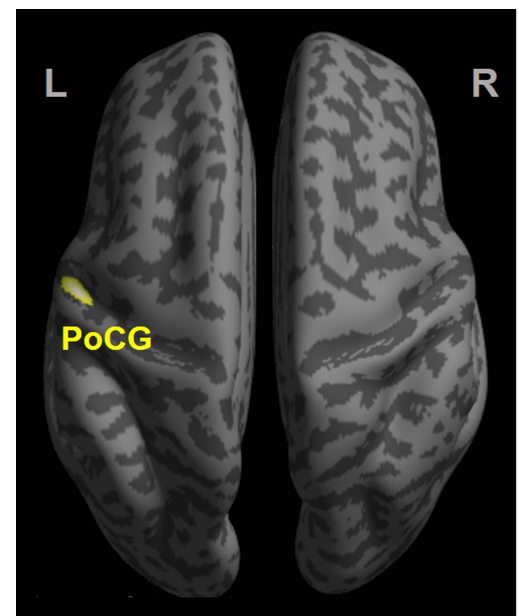

Fig. 2. Significantly reduced local cortical thickness in male patients with alcohol-dependence compared to male healthy controls (two sample ttest, uncorr. $\mathrm{p}<0.001$, cluster size: 74 voxels). L, left; R, right; PoCG, postcentral gyrus ( $\mathrm{x}, \mathrm{y}, \mathrm{z}=-48,-12,31)$.
$[5,24]$ were able to account for differences in education level and duration of smoking by using them as covariates in neuroimaging analysis. Based on these previous studies, we used education and duration of smoking as covariates during imaging processing to account for significant differences. However, compared to previous studies, the current study found decreased cortical thickness only in the PoCG despite the uncorrected thresholds. This discrepancy may stem from various factors such as the small sample size and/ or significant differences in education between two groups. Although the effects of education on cortical thickness are expected to be smaller than those of alcohol dependence, the addition of covariates to between-group differences into the statistical model may have reduced the statistical power to detect group effects on cortical thickness.

In the between-group comparison of total brain volumes, there were no differences in GM, WM, and CSF between the two groups. However, in the comparison of cortical thickness, the male ADPs 


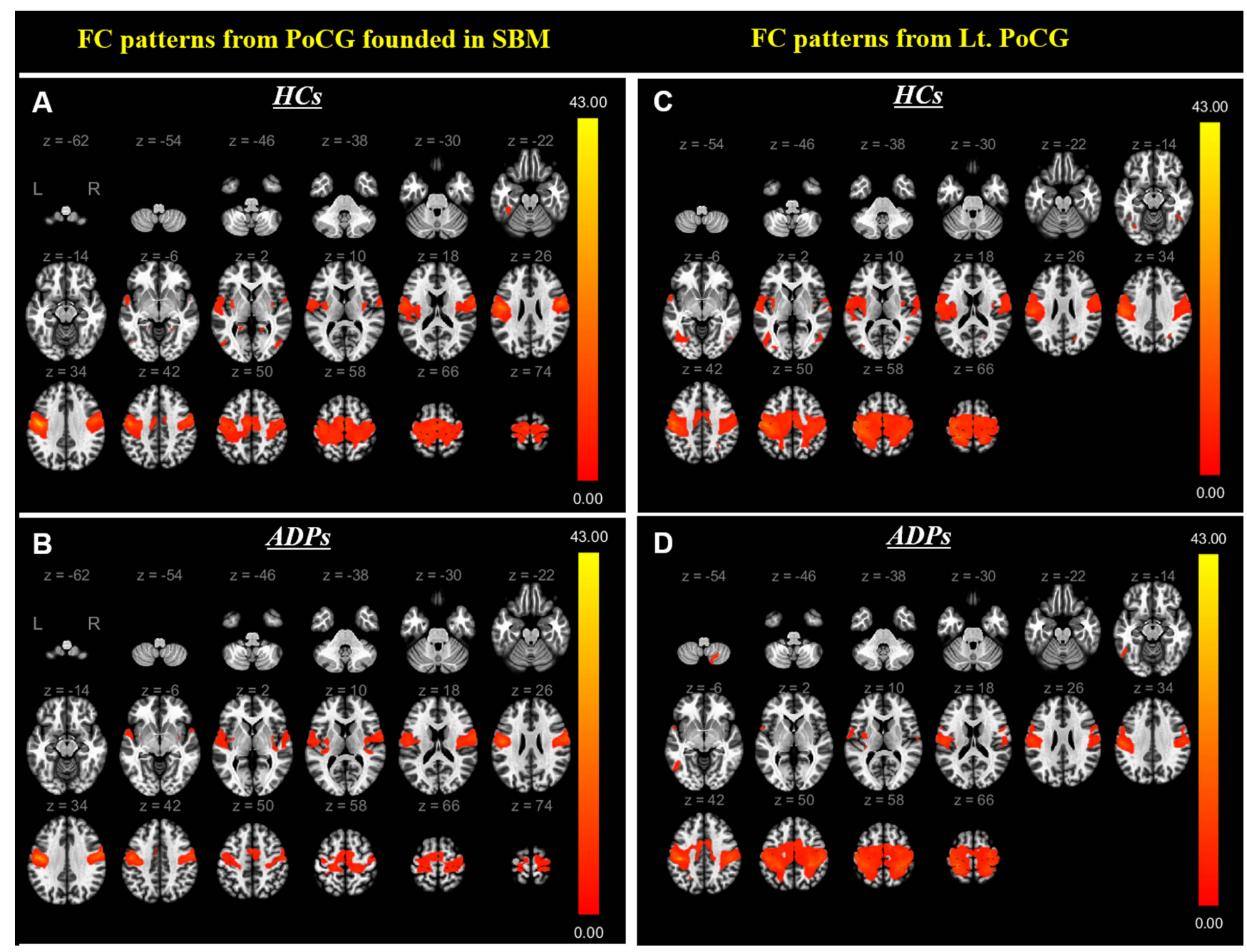

Fig. 3. The A and B panels showed FC patterns starting from localized regions with reduced cortical thickness in PoCG. The C and D panels showed FC patterns starting from the entire area of left PoGC (one sample t-test, voxel threshold: uncorr. $\mathrm{p}<0.001$, cluster threshold: $\mathrm{p}$-FDR $<0.05$ ). The color bars mean FC intensity. L, left; R, right; HCs, male healthy controls; ADPs, male patients with alcohol-dependence.

exhibited significantly reduced cortical thickness in PoCG. The PoCG, which contains the primary somatosensory cortex, is a significant brain region responsible for proprioception [25]. This region perceives various somatic sensations from the body, including touch, pressure, temperature, and pain [26]. Furthermore, neuroimaging studies were able to report various abnormalities in brain activation patterns $[27,28]$ and structures [29] in PoCG of ADPs. Morris et al. [29] reported decrease in PoCG neurite density of binge drinkers through perfusion studies. Furthermore, our findings confirmed reduced GM density of PoCG in terms of cortical thickness as our SBM analysis showed decrease in the distance from WM to GM. Since neuronal cell death may cause loss of function, this area was established as a seed area for assessing FC differences with reference to a previous neuroimaging study [30]. Our structural results showed limited brain area with signifi- cant differences despite a liberal threshold (uncorrected $\mathrm{p}<0.001$ ). This could be the result of various factors such as small sample size and/or significant differences in education level, which was used as a covariate with between-group differences in the statistical model. These fundamental issues of insufficient statistical power and/or other confounding factors may lead to substantial discrepancies compared to previous study [5] which showed widespread cortical thinning and GM volume loss with stringent statistical threshold (family-wide error).

In the assessment of brain FCs between male ADPs and male HCs, the results identified weaker and more restricted interregional activation synchrony within the connectivity network in addition to unique local areas in male ADPs compared to male HCs. These lower network-specific FCs have been generally associated with poorer performance and/or mood states in ADPs [31]. 


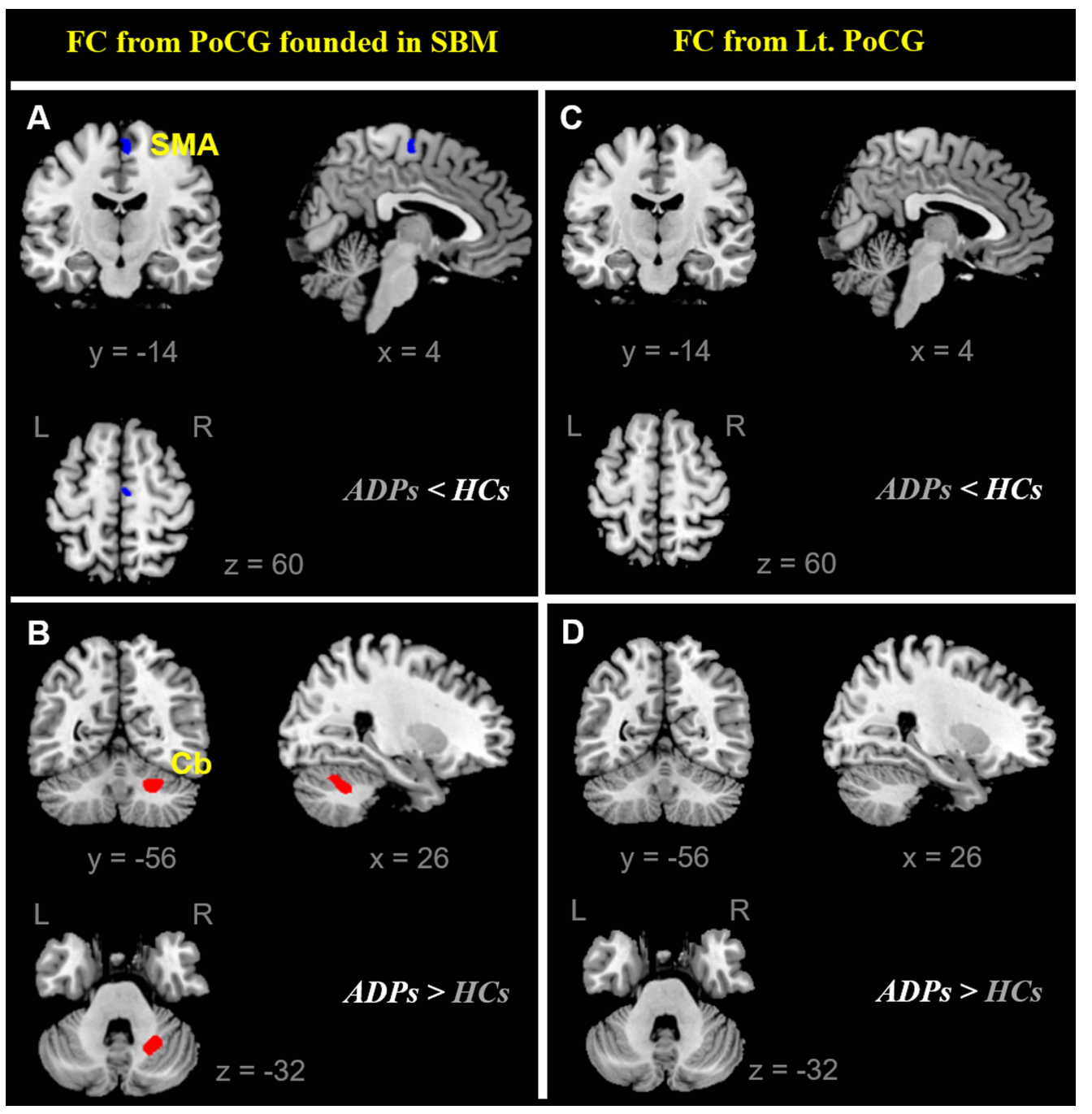

Fig. 4. The A and B panels shows significantly different FCs starting from localized regions with reduced cortical thickness in PoCG. C and D are panels with no significant differences in FC starting from the entire area of left PoGC. (two sample t-test, voxel threshold: uncorr. $\mathrm{p}<0.001$, cluster threshold: $\mathrm{p}-\mathrm{FDR}<0.05$ ). $\mathrm{L}$, left; R, right; HCs, male healthy controls; ADPs, male patients with alcohol-dependence; SMA, supplementary motor area; $\mathrm{Cb}$, cerebellum.

Table 3. Regions showing thickness-related functional connectivity differences between male patients with alcohol-dependence (ADPs) and male healthy controls (HCs) (voxel threshold : uncorr. $\mathrm{p}<0.001$, cluster threshold : $\mathrm{p}$-FDR $<0.05$ )

\begin{tabular}{|c|c|c|c|c|c|}
\hline \multirow{2}{*}{ Brain area } & \multicolumn{3}{|c|}{ MNI coordinates } & \multirow{2}{*}{ Cluster size } & \multirow{2}{*}{$\begin{array}{c}\text { Maximum } \\
\text { t-value }\end{array}$} \\
\hline & $\mathbf{x}$ & $\mathbf{y}$ & $\mathbf{z}$ & & \\
\hline Seed: postcentral gyrus (PoCG) & -48 & -12 & 31 & & \\
\hline \multicolumn{6}{|l|}{ ADPs } \\
\hline Right postcentral gyrus (Rt. PoCG) & 60 & -4 & 32 & 17,647 & 19.12 \\
\hline \multicolumn{6}{|l|}{$\mathrm{HCs}$} \\
\hline Right inferior occipital gyrus (Rt. IOG) & 54 & -76 & -2 & 223 & 4.59 \\
\hline Left middle occipital gyrus (Lt. MOG) & -54 & -76 & 2 & 186 & 4.81 \\
\hline Left lingual gyrus (Lt. LiG) & -22 & -52 & -2 & 184 & 5.42 \\
\hline Right lingual gyrus (Lt. LiG) & 22 & -46 & -2 & 153 & 4.69 \\
\hline Left fusiform gyrus (Lt. FuG) & -38 & -40 & -22 & 154 & 5.99 \\
\hline
\end{tabular}

We found reduced cortical thickness and weak FCs in male ADPs, demonstrating that neuronal cell death may cause neurofunctional abnormalities [30]. This finding could play a potential role in the pathophysiological understanding of alcoholism.
Male ADPs showed significantly lower and higher FCs in PoCGSMA and PoCG-Cb, respectively, compared to male HCs.

The SMA is embedded in motor circuits through its connections with the primary motor cortex, premotor cortex and cingulate 
Table 4. Distinct functional connectivity areas between male patients with alcohol-dependence (ADPs) and male healthy controls (HC) (voxel threshold : uncorr. $\mathrm{p}<0.001$, cluster threshold : $\mathrm{p}-\mathrm{FDR}<0.05$ )

\begin{tabular}{|c|c|c|c|c|c|}
\hline & \multicolumn{3}{|c|}{ MNI coordinates } & \multirow{2}{*}{ Cluster size } & \multirow{2}{*}{$\begin{array}{c}\text { Maximum } \\
\text { t-value }\end{array}$} \\
\hline & $\mathbf{x}$ & $\mathbf{y}$ & $\mathbf{z}$ & & \\
\hline \multicolumn{6}{|l|}{$\mathrm{ADPs}<\mathrm{HCs}$} \\
\hline Right supplementary motor area (Rt. SMA) & 4 & -14 & 60 & 424 & 4.95 \\
\hline \multicolumn{6}{|l|}{$\mathrm{ADPs}>\mathrm{HCs}$} \\
\hline Right cerebellum (Rt. Cb) & 26 & -56 & -32 & 752 & 6.59 \\
\hline
\end{tabular}

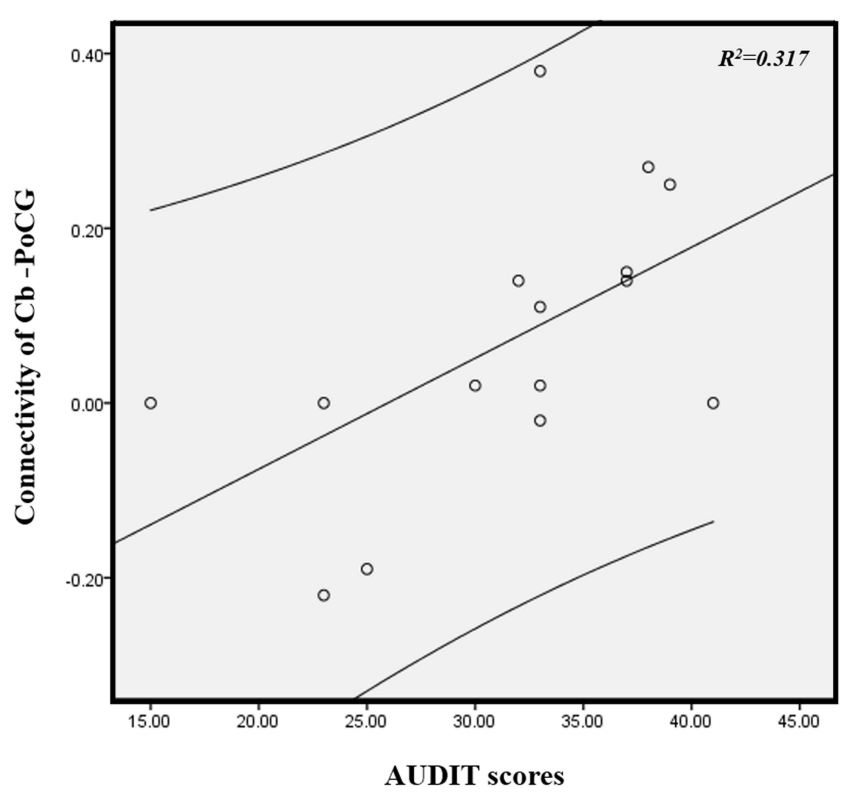

Fig. 5. The correlation between AUDIT scores and functional connectivities of Cerebellum $(\mathrm{Cb})$ linked to postcentral gyrus (PoCG) with reduced local cortical thickness in male patients with alcohol-dependence (Spearman's correlation, $\mathrm{r}=0.579, \mathrm{p}=0.024$ ). The curved line bands mean $95 \%$ confidence intervals. AUDIT, alcohol use disorders identification test.

cortex [32]. A large body of literature demonstrates [33-35] that processes involving the motor system may also contribute to inhibitory control, with successful inhibition entailing a decrease in motor system excitability. Additionally, a study showed that heavy drinking is related to higher impulsivity due to reduced response inhibition [36]. In a neuroimaging study [37], AUDIT scores were negatively related to functional network connectivity between the inferior occipital gyrus and the SMA.

The $\mathrm{Cb}$ has been considered to play an important role in the acquisition of motor coordination and the cognitive processes that control movement [38]. The $\mathrm{Cb}$ may also help augment cognitive processes originating in the cerebrum [39], such as memory [40], language production [41] and mental imagery [42]. Deficits of motor control and cognitive processes associated with abnormalities in cerebellar structure and function have been reported in alcoholism [43]. A neuroimaging study reported that the FC of PoCG-Cb was significantly greater in alcoholics than controls [31]. Interestingly, in our results, the increased FCs of PoCG-Cb had a positive correlation with AUDIT scores in male ADPs.

In summary, the abnormal FC of PoCG-Cb in male ADPs may arise from incoordination between the cognition and motor systems, as well as from poor adaptations to changes in sensory and motor input from alcohol-related $\mathrm{Cb}$ impairment.

There are some limitations in this study. First, the population of subjects consisted of only males with $15 \mathrm{ADMs}$ and HCs each. Therefore, future studies with a larger population and sex control are needed to increase the statistic power. Second, we could not rule out the potential effects of confounding factors of smoking and educational levels on the brain. However, we controlled for such factors as covariates to avoid confounding when we performed imaging processing for significant differences. Additionally, alcoholism may still be the dominant factor contributing to brain abnormalities between male ADPs and male HCs according to previous studies $[42,43]$.

This study demonstrated that the distinct brain cortical thickness reduction in PoCG and its association with FC changes correlated with clinical severities in male ADPs. Our results suggest that alcoholism may cause structural and functional network changes in the relevant brain regions. Our findings could be helpful for understanding the neural mechanisms of alcoholism and provide a new direction for its clinical treatment. In addition, these findings, along with previous multimodal neuroimaging studies [5, 44], provides additional information on structural and functional association in alcoholism.

\section{ACKNOWLEDGEMENTS}

This study was supported by the Research Program through the National Research Foundation of Korea (NRF) funded by the Ministry of Science and ICT (NRF-2017M3C7A1044367). 


\section{REFERENCES}

1. Maimaris W, McCambridge J (2014) Age of first drinking and adult alcohol problems: systematic review of prospective cohort studies. J Epidemiol Community Health 68:268-274.

2. Sebastiani G, Borrás-Novell C, Casanova MA, Pascual Tutusaus M, Ferrero Martínez S, Gómez Roig MD, García-Algar O (2018) The effects of alcohol and drugs of abuse on maternal nutritional profile during pregnancy. Nutrients 10:1008.

3. World Health Organization (2018) Global status report on alcohol and health 2018 [Internet]. World Health Organization, Geneva. Available from: https://apps.who.int/iris/ handle/10665/274603.

4. Day AM, Kahler CW, Ahern DC, Clark US (2015) Executive functioning in alcohol use studies: a brief review of findings and challenges in assessment. Curr Drug Abuse Rev 8:26-40.

5. Wang J, Fan Y, Dong Y, Ma M, Ma Y, Dong Y, Niu Y, Jiang Y, Wang H, Wang Z, Wu L, Sun H, Cui C (2016) Alterations in brain structure and functional connectivity in alcohol dependent patients and possible association with impulsivity. PLoS One 11:e0161956.

6. Shin SK, Kaiser EE, West FD (2021) Alcohol induced brain and liver damage: advantages of a porcine alcohol use disorder model. Front Physiol 11:592950.

7. Fein G, Di Sclafani V, Cardenas VA, Goldmann H, TolouShams M, Meyerhoff DJ (2002) Cortical gray matter loss in treatment-naïve alcohol dependent individuals. Alcohol Clin Exp Res 26:558-564.

8. Jernigan TL, Butters N, DiTraglia G, Schafer K, Smith T, Irwin M, Grant I, Schuckit M, Cermak LS (1991) Reduced cerebral grey matter observed in alcoholics using magnetic resonance imaging. Alcohol Clin Exp Res 15:418-427.

9. Pfefferbaum A, Lim KO, Zipursky RB, Mathalon DH, Rosenbloom MJ, Lane B, Ha CN, Sullivan EV (1992) Brain gray and white matter volume loss accelerates with aging in chronic alcoholics: a quantitative MRI study. Alcohol Clin Exp Res 16:1078-1089.

10. Harada T, Ishizaki F, Horie N, Katsuoka H, Nitta Y, Yamada T, Nitta K, Ito M (2011) Alcohol-induced persistent mild cognitive impairment with successful withdrawal from alcohol dependence--a case report. Hiroshima J Med Sci 60:11-13.

11. Yang K, Yang Q, Niu Y, Fan F, Chen S, Luo X, Tan S, Wang Z, Tong J, Yang F, Le TM, Li CR, Tan Y (2020) Cortical thickness in alcohol dependent patients with apathy. Front Psychiatry 11:364.

12. Sullivan EV, Marsh L, Mathalon DH, Lim KO, Pfefferbaum A (1996) Relationship between alcohol withdrawal seizures and temporal lobe white matter volume deficits. Alcohol Clin Exp Res 20:348-354.

13. Luciana M, Collins PF, Muetzel RL, Lim KO (2013) Effects of alcohol use initiation on brain structure in typically developing adolescents. Am J Drug Alcohol Abuse 39:345-355.

14. Sullivan EV, Marsh L, Mathalon DH, Lim KO, Pfefferbaum A (1995) Anterior hippocampal volume deficits in nonamnesic, aging chronic alcoholics. Alcohol Clin Exp Res 19:110-122.

15. Segall JM, Allen EA, Jung RE, Erhardt EB, Arja SK, Kiehl K, Calhoun VD (2012) Correspondence between structure and function in the human brain at rest. Front Neuroinform 6:10.

16. Sui J, Huster R, Yu Q, Segall JM, Calhoun VD (2014) Function-structure associations of the brain: evidence from multimodal connectivity and covariance studies. Neuroimage 102 Pt 1:11-23.

17. Wang J, Fan Y, Dong Y, Ma M, Dong Y, Niu Y, Jiang Y, Wang H, Wang Z, Wu L, Sun H, Cui C (2018) Combining gray matter volume in the cuneus and the cuneus-prefrontal connectivity may predict early relapse in abstinent alcohol-dependent patients. PLoS One 13:e0196860.

18. Lai KL, Niddam DM, Fuh JL, Chen WT, Wu JC, Wang SJ (2020) Cortical morphological changes in chronic migraine in a Taiwanese cohort: surface- and voxel-based analyses. Cephalalgia 40:575-585.

19. Chung W, Lim S, Lee S (2012) Why is high-risk drinking more prevalent among men than women? Evidence from South Korea. BMC Public Health 12:101

20. Flensborg-Madsen T, Becker U, Grønbæk M, Knop J, Sher L, Mortensen EL (2011) Alcohol consumption and later risk of hospitalization with psychiatric disorders: prospective cohort study. Psychiatry Res 187:214-219.

21. Rossetti MG, Patalay P, Mackey S, Allen NB, Batalla A, Bellani M, Chye Y, Cousijn J, Goudriaan AE, Hester R, Hutchison K, Li CR, Martin-Santos R, Momenan R, Sinha R, Schmaal L, Sjoerds Z, Solowij N, Suo C, van Holst RJ, Veltman DJ, Yücel M, Thompson PM, Conrod P, Garavan H, Brambilla P, Lorenzetti V (2021) Gender-related neuroanatomical differences in alcohol dependence: findings from the ENIGMA Addiction Working Group. Neuroimage Clin 30:102636.

22. Dahnke R, Yotter RA, Gaser C (2013) Cortical thickness and central surface estimation. Neuroimage 65:336-348.

23. Behzadi Y, Restom K, Liau J, Liu TT (2007) A component based noise correction method (CompCor) for BOLD and perfusion based fMRI. Neuroimage 37:90-101.

24. Filbey FM, Aslan S, Calhoun VD, Spence JS, Damaraju E, Caprihan A, Segall J (2014) Long-term effects of marijuana use on the brain. Proc Natl Acad Sci U S A 111:16913-16918. 
25. Kropf E, Syan SK, Minuzzi L, Frey BN (2019) From anatomy to function: the role of the somatosensory cortex in emotional regulation. Braz J Psychiatry 41:261-269.

26. Lloyd DM, McGlone FP, Yosipovitch G (2015) Somatosensory pleasure circuit: from skin to brain and back. Exp Dermatol 24:321-324.

27. Krienke UJ, Nikesch F, Spiegelhalder K, Hennig J, Olbrich HM, Langosch JM (2014) Impact of alcohol-related video sequences on functional MRI in abstinent alcoholics. Eur Addict Res 20:33-40.

28. Kamarajan C, Rangaswamy M, Tang Y, Chorlian DB, Pandey AK, Roopesh BN, Manz N, Saunders R, Stimus AT, Porjesz B (2010) Dysfunctional reward processing in male alcoholics: an ERP study during a gambling task. J Psychiatr Res 44:576590.

29. Morris LS, Dowell NG, Cercignani M, Harrison NA, Voon V (2018) Binge drinking differentially affects cortical and subcortical microstructure. Addict Biol 23:403-411.

30. Park SE, Kim BC, Yang JC, Jeong GW (2020) MRI-based multimodal approach to the assessment of clinical symptom severity of obsessive-compulsive disorder. Psychiatry Investig 17:777-785.

31. Müller-Oehring EM, Jung YC, Pfefferbaum A, Sullivan EV, Schulte T (2015) The resting brain of alcoholics. Cereb Cortex 25:4155-4168.

32. Luppino G, Matelli M, Camarda R, Rizzolatti G (1993) Corticocortical connections of area F3 (SMA-proper) and area F6 (pre-SMA) in the macaque monkey. J Comp Neurol 338:114140.

33. Duque J, Greenhouse I, Labruna L, Ivry RB (2017) Physiological markers of motor inhibition during human behavior. Trends Neurosci 40:219-236.

34. Bestmann S, Duque J (2016) Transcranial magnetic stimulation: decomposing the processes underlying action preparation. Neuroscientist 22:392-405.

35. Quoilin C, Derosiere G (2015) Global and specific motor inhibitory mechanisms during action preparation. J Neurosci 35:16297-16299.

36. Ahmadi A, Pearlson GD, Meda SA, Dager A, Potenza MN, Rosen R, Austad CS, Raskin SA, Fallahi CR, Tennen H, Wood RM, Stevens MC (2013) Influence of alcohol use on neural response to Go/No-Go task in college drinkers. Neuropsychopharmacology 38:2197-2208.

37. Vergara VM, Liu J, Claus ED, Hutchison K, Calhoun V (2017) Alterations of resting state functional network connectivity in the brain of nicotine and alcohol users. Neuroimage 151:4554.

38. Ito M (1993) Movement and thought: identical control mechanisms by the cerebellum. Trends Neurosci 16:448-450; discussion 453-454.

39. Leiner HC, Leiner AL, Dow RS (1993) Cognitive and language functions of the human cerebellum. Trends Neurosci 16:444-447.

40. Appollonio IM, Grafman J, Schwartz V, Massaquoi S, Hallett M (1993) Memory in patients with cerebellar degeneration. Neurology 43:1536-1544.

41. Petersen SE, Fiez JA (1993) The processing of single words studied with positron emission tomography. Annu Rev Neurosci 16:509-530.

42. Ryding E, Decety J, Sjöholm H, Stenberg G, Ingvar DH (1993) Motor imagery activates the cerebellum regionally. A SPECT rCBF study with 99mTc-HMPAO. Brain Res Cogn Brain Res 1:94-99.

43. Sullivan EV, Rosenbloom MJ, Deshmukh A, Desmond JE, Pfefferbaum A (1995) Alcohol and the cerebellum: effects on balance, motor coordination, and cognition. Alcohol Health Res World 19:138-141.

44. Beck A, Wüstenberg T, Genauck A, Wrase J, Schlagenhauf F, Smolka MN, Mann K, Heinz A (2012) Effect of brain structure, brain function, and brain connectivity on relapse in alcohol-dependent patients. Arch Gen Psychiatry 69:842-852. 